\title{
Extending Epigenesis: From Phenotypic Plasticity to the Bio-Cultural Feedback
}

\author{
Paolo D'Ambrosio ${ }^{1}$ and Ivan Colagè ${ }^{1 *}$
}

${ }^{1}$ Pontifical Antonianum University, Via Merulana $124-00185$, Rome, ITALY

*Corresponding Author: e-mail i.colage@antonianum.eu; Tel. +39 067037 3502; Fax. +39 0670373604.

\begin{abstract}
The paper aims at proposing an extended notion of epigenesis acknowledging an actual causal import to the phenotypic dimension for the evolutionary diversification of life forms. Section 1 offers introductory remarks on the issue of epigenesis contrasting it with ancient and modern preformationist views. In Section 2 we propose to intend epigenesis as a process of phenotypic formation and diversification a) dependent on environmental influences, b) independent of changes in the genomic nucleotide sequence, and c) occurring during the whole life span. Then, Section 3 focuses on phenotypic plasticity and offers an overview of basic properties (like robustness, modularity and degeneracy) that allows biological systems to be evolvable - i.e. to have the potentiality of producing phenotypic variation. Successively (Section 4), the emphasis is put on environmentally-induced modification in the regulation of gene expression giving rise to phenotypic variation and diversification. After some brief considerations on the debated issue of epigenetic inheritance (Section 5), the issue of culture (kept in the background of the preceding sections) is considered. The key point is that, in the case of humans and of the evolutionary history of the genus Homo at least, the environment is also, importantly, the cultural environment. Thus, Section 6 argues that a bio-cultural feedback should be acknowledged in the "epigenic" processes leading to phenotypic diversification and innovation in Homo evolution. Finally, Section 7 introduces the notion of "cultural neural reuse", which refers to phenotypic/neural modifications induced by specific features of the cultural environment that are effective in human cultural evolution without involving genetic changes. Therefore, cultural neural reuse may be regarded as a key instance of the bio-cultural feedback and ultimately of the extended notion of epigenesis proposed in this work.
\end{abstract}

Keywords: bio-cultural feedback, cultural neural reuse, epigenesis, evolvability, phenotypic plasticity, neural plasticity.

\section{Introductory Remarks}

As some authors have pointed out (see for instance Jablonka \& Lamb 2002), the current view of epigenesis can be traced back to the studies on the constitution of living beings undertaken by Aristotle. Briefly speaking, Aristotle inferred from empirical investigations a causal theory that could have accounted for both the contingency and the finality displayed in the biological realm. Organisms' internal organization is seen as an integration of parts gradually constructed through qualitative changes during development: organisms are literally generated (genesis) by progressively building structures upon (epi) less differentiated ones. Aristotle saw individual differences in specimens as accidents or as being contingently brought about whereas, provided that no factors come to disrupt the process (again occasionally), the final outcome, that is, the formation of a mature individual, is necessarily accomplished. This occurs because of a meta-empirical, permanent principle: the essence, the form, or the biological species 
"typologically" intended, which would drive the developmental process from the start, thus reaffirming itself through diversity.

For the sake of introducing the main issues discussed in the present paper, it is also worth recalling that for Aristotle living beings are by definition capable of autonomous action, as they are provided with a psyche; instead, inanimate physical entities are essentially passive. The process of development and growth, implying adjustment to environmental fluctuations, represents a modality common to both animals and plants, in which such a distinctive potentiality of self-driven action is realized.

In the context of eighteenth-century pre-modern biology, a weighty controversy took place between the advocates of epigenesis and the proponents of the so-called "preformationism", according to which a fully-formed miniature of the adult organism was believed to be already enclosed in a germ cell, and development would have merely consisted in the growth of such a miniature being. Note that both epigenesis and prefomationism were framed in the then still dominating essentialist/fixist view of biological species. The passage to modern biology, with the spread of evolutionary ideas and the rejection of fixism, can be regarded as the first (and probably the sole, until now) true revolutionary paradigm shift in the study of living beings. One of the main reasons is the following: once species are recognized as being able to trans-form one into another through time, variation can no longer be negligible as something occurring with little if any substantial significance (as "accidental" sensu Aristotle); rather, the modalities of diversification and the origin of individual differences become precisely the major explananda.

On this background, the goal of the present paper is to propose a more extended notion of epigenesis for explaining the diversification of phenotypes in relation to environmental influences. The novelty of our proposal consists in acknowledging an actual causal role of the phenotypic dimension for the arising of evolutionary diversification of life forms. This implies that phenotypes 1) should not be considered as mere mechanist outcomes of the underlying bio-molecular factors (be they genetic or not), and 2) should not be regarded as relevant for evolutionary dynamics only in relation to natural selection but also as a fundamental source of variation per se.

To this aim, after defining the criteria of our extended notion of epigenesis (Sect. 2), focusing on phenotypic plasticity and evolvability (Sect. 3), discussing cases of environmentallyinduced phenotypic diversification (Sect. 4), and assessing epigenetic inheritance (Sect. 5), we will address the issue of bio-cultural feedback (Sect. 6). Indeed, the role that culture can play in inducing phenotypic diversification can be regarded, in our view, as an extreme instance of the actual causal import of the phenotypic dimension for the evolutionary diversification of life forms that, however, still falls within our extended notion of epigenesis. Contextually, we choose to focus on the brain substrates for reading ability (Sect. 7) as several studies in the last two decades have gathered clear evidence for the effectiveness of such a cultural practice in shaping the brain phenotype.

\section{Transmutation of Forms: Phenotypic Variation, Diversification, and Complexification}

As M. Pigliucci pointed out, referring to a comment by K. Popper, the mid-twentieth century Synthetic Theory of Evolution does a good job in explaining the evolution of genes, whereas the other main issue that evolutionary biology is called upon to explain remains unexplored, that is, the transmutation of forms (Pigliucci 2009). Addressing this main issue means to inquire into the causal factors responsible for variation, diversification and complexification of phenotypes in evolution. 
Darwin clearly stated that natural selection "can do nothing" without the arising of variation in the progeny (Darwin 1859, Ch. 4), and that selection has "no relation whatever" to the primary cause of variation production (Darwin 1868, vol. II, p. 321). The interactional dynamics underlying selection can determine which phenotypic variants get fixed and diffused in populations, but cannot "create" those variants in the first place; "natural selection may account for the survival of the fittest but cannot explain the arrival of the fittest" (de Vries 1905, p. 826). On the other hand, phenotypic change cannot stem solely from spontaneous alterations in the inherited genome, given that a considerable amount of genetic mutation does not lead to appreciable phenotypic variants, whereas many phylogenetically related organisms display remarkable phenotypic differences, which are apparently not causally linked to small differences in their specific DNA sequences. It has been realized that the detailed structural and functional configuration of a phenotype cannot be a priori or mechanistically deduced on the sole basis of a genome (Schlichting and Pigliucci 1998). Briefly speaking, genetic evolution does not coincide with, or follow the same rate as, phenotypic evolution. In other words, the diversification in time of life forms (see Sect. 1) cannot be investigated only at the genetic level, as this would surreptitiously reintroduce a subtle version of "preformationism", according to which the development of the mature organism is "preformed" in the genome (Oyama et al. 2001).

The quest for the origination of phenotypic diversification is currently undertaken by reconsidering the causal connections between evolution and development (Darwin 1859, Ch. 13; Amundson 2005, Ch. 9) in the wide-ranging perspective of Evolutionary Developmental Biology (Evo-Devo) (Burian et al. 2000; Müller 2007; Collins et al. 2007). Indeed, the initial "programming" deriving from the inherited genome turns out to be significantly rearranged in the course of development. The notion of developmental reprogramming (Arthur 2000) captures the idea that the activity of gene clusters or networks relative to the construction of organisms' internal organization can be differently regulated from the early stages of embryo formation. In this respect, Waddington coined the term "epigenetics" for referring to the interactions between genes and their products giving rise to different phenotypic outcomes (Waddington 1942a; Waddington 1968). Various phenotypes can be produced starting from a certain genome, as development can take alternative trajectories because of different environmental conditions, as stated in terms of norms of reaction (Schmalhausen 1949, Ch. 1). Hence, attention is turned to the complex of developmental processes correlating a genotype to a phenotype, or to what Waddington called the epigenotype (Waddington 1942b), featuring the interactions between gene networks, their products, and environmental influences (Gilbert 2000).

In the last decade, several definitions of the word "epigenetics" have been put forward (Ptashne 2007; Ho and Burggren 2010). The term is usually referred to molecular mechanisms modulating DNA independently of alterations in the DNA sequence, or to the regulation of gene expression exerted by pathways of interactions between proteins and/or RNAs producing appreciable phenotypic outcomes (Allis and Jenuwein 2016). It has also been stressed that epigeneticallyinduced modifications are not confined to developmental stages and are stable across the entire lifespan of individuals (e.g. Daxinger and Whitelaw 2012). "(E)pigenetics may be defined as the study of any potentially stable and, ideally, heritable change in gene expression or cellular phenotype that occurs without changes in Watson-Crick base-pairing of DNA" (Goldberg et al. 2007, p. 635). Some authors focus on epigenetic inheritance, i.e. inheritable mechanisms allowing the transmission of altered phenotypes across lineages of cells having an identical genotype ("cellular heredity", "intergenerational epigenetic inheritance"), or across lineages of 
organisms ("transgenerational epigenetic inheritance") (Jablonka and Lamb 2006a; Burggren 2014; Richards 2006; Probst et al. 2009).

Here, we use the term epigenesis (as distinct from "epigenetics") for referring broadly to the generation of phenotypes and of phenotypic diversity (disease states and functionally-impaired variants included), a process that involves the incidence of environmental factors throughout the life cycle. Such a broad conception of epigenesis is consistent with the original meaning of the word, which can be traced back to Aristotle (see Sect. 1). However, we stress that the construction of phenotypes is accomplished by virtue of external inputs and resources, and that environmental factors also play a crucial role in the emergence and evolution of organisms' diversity. Therefore, our position is close to the perspective of "Ecological Developmental Biology" (Eco-Devo) (Gilbert 2001; Sultan 2007; Gilbert and Epel 2009; see also D’Ambrosio 2016), yet we emphasize the fact that the external (and changing) environmental conditions continue to be effective across the whole life cycle, beyond developmental stages.

Now, within such an extensive view of epigenesis, epigenetics is meant to refer to molecular mechanisms not involving alterations in the DNA sequence, which - be they inheritable or not should be empirically attestable as being affected, or at least triggered, by environmental factors. In other words, we would put together the dependence on environmental parameters and the independence from alterations in the genomic nucleotide sequence as a demarcation criterion for "epigenic" processes, of which the underlying bio-molecular mechanisms represent a subset (see Fig. 1). Consequently, our extended notion of epigenesis (or of "epigenic" processes) encompasses any process of generation of phenotypic variation irrespective of the underlying bio-molecular mechanisms, provided that such processes are a) dependent on environmental influences, b) independent of changes in the genomic nucleotide sequence, and c) that they can occur during the whole life span. Note that such an extended conception of epigenesis takes for granted that the generation of phenotypic variation certainly involves those bio-molecular mechanisms pertaining to the epigenetic level (see Fig. 1). However, according to the main goal of this paper (Sect. 1), the notion of epigenesis so conceived is meant to emphasize that the phenotypic dimension has a significant causal import per se, and that the production of phenotypic variation is not only an outcome of underlying bio-molecular mechanisms.

\section{Evolvability and Phenotypic Evolution}

The production of phenotypic diversification is increasingly understood as rooted in organisms' plasticity. Phenotypic plasticity has been defined as the ability of an organism to react to environmental cues through changes in form, behaviour or rate of activity (WestEberhard 2003, Ch. 3). Although the term "developmental plasticity" is often used for referring to the production of alternative phenotypic forms (polyphenism) in response to changing environmental conditions (see also Fusco and Minelli 2010; Moczeck et al. 2011), phenotypic plasticity may well be not relegated to developmental stages alone. The potential to produce selectable phenotypic variation has been intended in terms of organisms' evolvability (Kirschner and Gerhart 1998). In our view, evolvability stems from the general capacity of finding appropriate trade-offs between openness and closure to the environment (see also Auletta et al. 2015). Organisms are shielded against perturbations which may endanger homeostasis and be disruptive; especially in animals, development itself is largely buffered against external perturbations by means of internal constraints insuring the stability of the process. On the other hand, organisms are also open and exposed to the environment, and dealing with pressures 
coming from the outside presupposes the capability to actively respond to fluctuating parameters or stimuli.

Three features of organisms underlie their being "evolvable": robustness, modularity and degeneracy. Robustness (Hammerstein et al. 2006) ensures the persistence of structural and functional phenotypic features in spite of genetic mutations or environmental fluctuations. For example, robustness allows for the accumulation of "cryptic" genetic variation (Masel and Siegal 2009), which does not have immediate effects on the phenotype but can support future production of (possibly adaptive) phenotypic variants when particular environmental conditions and/or genetic backgrounds become available (Wagner 2005). Modularity (Schlosser and Wagner 2004) refers to the fact that organisms are composed of distinguishable (but not totally separated) sub-systems that can also evolve, at least in part, independently of each other. It promotes phenotypic complexification and progressive specialization as specific functions are deferred to relatively independent sub-systems. Together with robustness, an increased modularity can also lower the probability of deleterious variants in pre-existing stable structures (see also Wimsatt and Shank 2004).

However, organisms are not just assemblies or collections of more and more specialized sub-systems, each being sufficient to subserve one particular function. Darwin was likely right in thinking of progressive evolution as characterized by increasing distribution of tasks, or "division of labour" (Darwin 1868, vol. I, p. 9-10), which allows organisms to control more aspects of the surrounding environment (see also Schmalhausen 1949, Ch. 3). However, this is usually accomplished together with forms of articulated connectivity between subsystems; in other words, increased modularity and specialization is usually accompanied by connectedness (WestEberhard 2003, Ch. 4). Biological complex systems are those revealing an interplay between functional specialization or segregation, and functional integration (Edelman and Gally 2001; Tononi et al. 1999).

So, biological systems can be considered as networks of modules. From this viewpoint, biological structures at several levels (from genes or enzymes, to organs or behavioural patters, etc.) can serve as modules in functional networks. In general, a function can be fulfilled by a whole network, and not by single isolated modules, whereas each module has its own specific operating modality. A module, while maintaining its working modality, can be part of different functional networks and, consequently, contribute to the fulfilment of more than one function (Fig. 2). This leads to the third feature ensuring evolvability: degeneracy. Degeneracy has been defined as the capability of structurally different elements to yield the same or different functional outputs depending on the context (Edelman and Gally 2001). It is worth stressing that degeneracy is not in contradiction with modularity, provided the latter is not understood as "massive modularity". (Indeed, we do not conceive biological modules as "domains of information" that are mutually impermeable and related to single functions, but as elements identifiable as pertaining to functional networks or domains.)

Degeneracy represents another ubiquitous characteristic of biological systems at all scales: gene regulatory sequences, proteins having no apparent structural, physiological or evolutionary relationship, intercellular signalling, pathways of organismal development, immune responses, connectivity in neural networks, neuronal mechanisms underlying synaptic plasticity, and behavioural repertoires. Degeneracy has a remarkable adaptive value since recurring to different but functionally equivalent solutions can be extremely useful for dealing with the "unexpected" (see also Auletta et al. 2008), not only in the case of potentially lethal genetic mutations but also for counterbalancing environmental challenges. In other words, degeneracy improves robustness to perturbation and increases flexibility to changing 
environments (Mason 2014). Thus, degeneracy may be seen as an important factor of evolvability specifically intended as the propensity to produce phenotypic innovations (Whitacre 2010).

Nevertheless, exploiting and managing the potential of internal degenerate structures, so as to respond to environmental demands, does not mean that organisms can directly sort out the most adequate option, put to work the "right strategy", and change accordingly. Rather, what seems to happen is an unguided or "blind" search between alternatives, some of which are (selectively) engaged for playing adaptive functional roles. As has been pointed out with regards to neuro-functional development and scattered brain areas supporting a human-specific cognitive ability like language, an active search is implemented implying the testing of multiple neural partnerships "to identify functionally adequate options, in some cases multiple alternative possibilities, leading to a degenerate functional network that can be modulated depending on circumstances and task demands" (Anderson and Finlay 2014, p. 12). This point will be relevant for our discussion of cultural neural reuse in acquiring reading skill (Sect. 7).

In concluding this section, we emphasize that robustness, modularity and degeneracy are features of biological systems ascertainable not only at the genetic or molecular level, but also at the macroscopic phenotypic level (including behavioural patterns). This contributes to strengthen our point about the causal effectiveness of the phenotypic dimension per se in the production of evolutionary diversification of forms.

\section{Environmental Induction of Phenotypic Changes}

The accomplishment of ordinary development strongly relies on biotic and abiotic environmental features, like gravitation, temperature, substances consumed for nutrition, interactions with other organisms, and so forth. Correct formation and preservation of functional structures depend on the incidence of a number of external conditions. Hence, alterations in these conditions can produce remarkable phenotypic modifications, which may or may not have viable results. Moreover, external factors are involved in other life-cycle processes - such as metamorphosis, regeneration, developmental symbiosis, sexual-asexual progression, and learning (Gilbert 2001) - that involve deep changes in organisms' functional organization and from which phenotypic modifications can arise as well. Finally, cultural components of the environment in human societies can exert a distinctive influence on phenotype formation and differentiation, as will be better clarified in Sections 6 and 7 below. Here, we report some case studies highlighting environmental induction of phenotypic changes.

Gravity, the most basic physical force/gradient on Earth, affects cell polarity and cell-to-cell interactions (e.g., Cove 2000). Experimental surveys have explored the modifications that altered gravity can induce on cell cytoskeleton and on cell development during embryogenesis in different animals (Crawford-Young 2006). Experiments performed on rats aboard the space shuttle Columbia have shown that the formation of mammalian motor system during postnatal development is affected by gravity levels (Walton et al. 2005). A research project is being developed (Auletta et al. 2012; Adell et al. 2014) with the aim of ascertaining the effects of microgravity conditions on the establishment of the anterior-posterior axis in planarians ( $S$. mediterranea), and its re-specification in relation to planarians' unique regenerative capabilities allowed by a widespread population of neoblasts, i.e. pluripotent stem cells (Saló et al. 2009).

Pressures exerted by tension forces affect animals' bone formation by activating bone morphogenetic proteins (BMPs) (Sato 1999). The development of jaw size and shape may be particularly intriguing as it involves mechanical tension in relation to food grinding, and consequent modifications due to diet. During normal mandibular advancement, mechanical- 
tension stress is mediated by a paracrine factor (indian hedgehog) transducing mechanical signals for stimulating chondrocyte proliferation and growth of mandibular cartilage (Tang et al. 2004). In the case of humans, reduction in masticatory stress is acknowledged as having influenced the evolution of the skull. On the basis of experimental data attesting differential jaw structuration and functionality depending on hard-food or soft-food diets, it has been suggested that both decrease in jaw dimension and increase in occlusal variation displayed by modern human beings do not derive from genetic alterations but from factors relative to cultural environment, namely, the transition from pre-industrialized food to modern soft diet, which requires less chewing force and time. This prompted the so-called "masticatory-functional hypothesis" for craniofacial changes (Corruccini 1984; Varrela 1992; Varrela 2006).

So-called "same genotype-diverse phenotypes" cases in several species seem to be due to diet changes. Variable diet can influence levels of DNA methylation, a crucial mechanism for regulating gene expression whose alterations during development can produce diversified phenotypic outcomes. Experimentally feeding pregnant rats with methyl donor supplements led to increased levels of fetal DNA methylation that blocked the transcription of the agouti gene and brought about the formation of morphologically-atypical progeny exhibiting dark hair and smaller size, as well as modified lipid metabolism (Waterland and Jirtle 2003). In natural conditions, the marked morphological and behavioural differences of genetically identical working vs. queen honey bees ( $A$. mellifera) are due to specialized diet during development: larvae of future queens are raised on royal jelly, and this seems correlated with different levels of DNA methylation in queens and workers respectively (Lyko et al. 2010).

Differential DNA methylation can also be induced by other external factors relative to intraspecific behaviours in the course of post-embryonic development. For instance, it has been demonstrated that specific postnatal maternal care in rats (grooming and licking as well as arched-back nursing) brings about differential methylation in the promoter region of the gene expressing glucorticoid receptors in the hippocampus (Weaver et al. 2004), thus altering the hypothalamic-pituitary-adrenal responses to stress in the offspring (Weaver 2007). In brief, mice receiving more maternal care after birth will develop more glucorticoid receptors, and will deal with stress better than individuals that received less care (see Gilbert 2006, Ch. 22).

In general, behavioural variation and polymorphism in several taxa have been shown to arise in connection with modulation of neural networks, or with changes in the amount of neurotransmitters in these networks, accompanying key passages in the life cycle such as the onset of sexual maturity, metamorphosis stages in insects and amphibians, or "distribution of tasks" in social insects. For instance, locusts like those belonging to the species $S$. gregari alternatively display a solitary and a gregarious phenotypic configuration with striking differences not only in morphology but also in physiology and behaviour. The shift from solitary to gregarious is driven by environmental conditions and may occur over time scales ranging from hours - sufficient for some behavioural modifications - to the passing of generations leading to full change in form; long-lasting trans-generational modifications seem also to be dependent on maternal influence over embryonic development (Bouaïchi et al. 1995). Phase transitions are accompanied by changes in the amount of neuroactive substances produced in both brain and thoracic nerve cord (Rogers et al. 2004).

Changes in environmental conditions may also induce phenotypic modifications at the level of interspecific interactions, from parasitism to mutualism (Sapp 1994, 2003). The role of evolution by symbiogenesis in the appearance of phenotypic innovations and macroevolutionary transitions has been widely acknowledged (e.g., Margulis and Fester 1991). A remarkable case is the role played by the impressive number of bacteria hosted in the lower 
gastrointestinal tract of mammals (Hooper et al. 1998, 2002). Specific autochthonous microorganisms like $B$. fragilis contribute to mediating the correct cellular development of the mammalian immune system (Mazmanian et al. 2005). Host-bacterial associations were shaped and fine-tuned in the course of evolution so to give rise to functionally-interdependent and highly stable biological systems: as has been noticed (Gilbert 2006, Ch. 22), hosts evolved not only to tolerate commensal microorganisms but to require them for the formation and preservation of fully functional individuals.

The expression of brain-derived neurotrophic factor (BDNF) - which promotes neurogenesis, neurodifferentiation, and axonal growth and connectivity, and is a major determinant of neuroplasticity (Murray and Holmes 2011; Calabrese et al. 2014) - depends essentially on external stimuli, as the exposure to an enriched environment may show (Vazquez-Sanroman 2013).

Although regarding very different biological phenomena and mechanisms, all these cases can be included in the extensive epigenic view delineated in Section 2 (see also Fig. 1). They testify to the direct influence of environmental factors on the construction of phenotypes and to "environmental induction" of phenotypic changes. Importantly, some cases regard processes occurring beyond development, and some other cases regard processes involving cultural factors in human societies.

Before addressing the implications of cultural factors for the proposed extended conception of epigenesis, especially as far as post-developmental stages are concerned, let us add some words on the debated topic of epigenetic inheritance.

\section{Assessing Epigenetic Inheritance}

Given that variation production is significantly induced by changes in environmental conditions, does such a state of affairs also imply consequences for the processes of variation transmission? Is it possible to speak in terms of a system of inheritance consisting in the transmission of an epigenetic endowment rather than of a genetic one?

The main ground for epigenetic inheritance may be seen in the fact that certain regulatory patterns of gene expression "survive" the early stages of development, are preserved during fertilization or gametogenesis, and can be therefore passed to next generations of cells (Petronis 2010; Dupont et al. 2009). The best examples of epigenetic inheritance are found in plants since "in contrast to most animals, where the germline is segregated off quite early in embryogenesis, the germline of plants is repeatedly derived from somatic cells. Consequently, epigenetic states established during the development of the plant soma may sometimes persist and be transmitted to the next generation" (Jablonka and Lamb 2006b, p. 359).

Now, in order to assess transgenerational epigenetic inheritance, implying the transmission of altered phenotypes across generations of organisms (Sect. 2), and its weight for adaptive evolution, at least two issues are worth considering. One is to ascertain the transmission of altered phenotypic characters in absence of the environmental stimuli or conditions which initially induced them, and the number of generations in which individuals continue to display those characters. This can be labelled as the "external" requirement. The "internal" requirement is in a sense more radical: are epigenetic channels of inheritance really non-genetic? If the hereditary transmission of patterns of regulation of gene expression results accompanied by, or even dependent upon, some kind of inherited genetic alterations, then we are likely in the presence of additional modalities of variation transmission as specific cases pertaining to the same general system of inheritance. One might speak in terms of nonMendelian (rather than "non-genetic" or "extra-genetic") forms of trans-generational 
inheritance involving the transmission of heritable epigenetic regulatory factors. For instance, this terminology has been used in another case study regarding RNA-dependent transmission of altered phenotypes in mice (Liebers et al. 2014): heritable RNA molecules affecting gene expression during development (RNA-mediated methylation) are showed to be responsible for a certain perpetuation of remarkable phenotypic modifications (paramutations) that persist for a limited number of generations. As long as non-Mendelian forms of inheritance coexist with "traditional" genetic inheritance, another issue to be clarified is represented by the relationships that could be established between heritable epigenetic factors and gene products differently expressed because of spontaneous mutations, including large-scale genetic rearrangements, which can occur in the passage from one generation to the next.

In brief, the main point seems to be again in appraising the causally-effective links between a genetic background and the regulating epigenotype differently modulated through interactions engaged with the external environment. Let us consider the case of environmentally-derived developmental changes in sensorimotor systems including differential activation of mirror neurons (Ferrari et al. 2013). The authors seem quite convergent on our line of interpretation: epigenetic mechanisms are intended as regarding "DNA's differential expression of proteins as a consequence of environmental influences (at the cellular, tissue, and whole organism levels)" (p. 451). They also stress that environmental conditions can affect neuronal connections and the stabilization of functional brain architecture by significantly impinging on epigenetic regulatory mechanisms, especially in postnatal development. Along this line, the evolutionary establishment of the mirror neuron system with its distinctive flexibility (or potentiality of being differently modulated) is explained in terms of stabilizing natural selection of environmentally-induced phenotypic changes. Postnatal development can be canalized along specific trajectories as long as certain environmental (in this case social) conditions are preserved (and this may be an example of what we have named above an "external requirement" for epigenetic inheritance to have an actual impact on adaptive evolution). In other words, the establishment of the mirror neuron system might provide a beautiful example of how epigenetic processes have a result which is crucial for determining stable phenotypic modifications on the basis of an evolutionarily established species-specific genome - modifications that remain nevertheless not transmissible to next generations in absence of triggering external stimuli, at least as far as has been possible to ascertain on empirical grounds up to now. As Ferrari and colleagues also point out, individual (sensorimotor) experience is often fundamental for "guiding developmental trajectories" and for shaping "the raw material provided by genes" according to contexts of social interactions (p. 454-455). To our mind, the intriguing point seems that a remarkably plastic sub-system has been selected and genetically transmitted, and it is this genetically transmitted sub-system that can be epigenetically re-oriented and even acquire different functional properties as new stimuli or challenging tasks are provided by environmental contexts.

Finally, let us recall the aforementioned distinction between epigenesis broadly intended, epigenetics, and epigenetic inheritance (Sect. 2 and Fig. 1). According to the view proposed here, epigenic processes do not concern only changes in levels of gene methylation or in chromatin structure, which may be shown to be inheritable. Be they biologically transmissible or not to subsequent generations, phenotypic modifications generally due to epigenesis occur because of the influence of environmental factors, and thanks to plasticity allowing organisms to react appropriately, for instance by rearranging and/or differently regulating their internal resources (see also Sect. 3 and Fig. 2). 


\section{Bio-Cultural Feedback}

In the case of human beings, the relevant environment is composed of both physical (biotic and abiotic) and cultural features. To what extent may cultural features affect human phenotypic diversification and evolution? And how may different cultural practices influence the biological constitution of human beings?

In trying to address these questions, let us begin with a quick look at some aspects of the evolutionary history of our genus. At least three major trends can be acknowledged in Homo evolution: complexification of the social dimension, technological progress, and encephalization (Bickerton 2014; Manzi 2012; Schick and Toth 2006; Colagè 2016). Exploiting a scavenging niche required advanced technological skills and enhanced social cohesion for early hominins to compete with other specialized scavengers/predators. At the same time, feeding on carcases ensured an increase in diet quality and the assimilation of fatty acids. This made it possible to sustain the process of encephalization characteristic of the genus Homo. Larger brains could in turn support further developments in technology and social relationships. Such an augmented social and technological lifestyle implied more and more sophisticated communicative skills crucial for both holding together the groups and ensuring the transmission of technology from generation to generation. It seems reasonable to think that these three major trends eventually led to the emergence of articulate language and teaching (and their mutual interrelations).

Indeed, the correlation between evolution of language and that of tool making is more and more acknowledged (e.g. Stout 2011; Stout and Chaminade 2012). Recent experiments performed on contemporary humans learning Oldowan tool making (Morgan et al. 2015) unveil the links between language, technology and teaching strategies. These results show that, given the relative difficulty of acquiring and transmitting the required skill, mastering Oldowan stone tools is enhanced by teaching, and in particular by language-assisted teaching, whereas forms of learning based on imitation or emulation ("observational learning") are much less effective. Thus, this kind of tool making would have plausibly favoured the diffusion of forms of verbal teaching granting higher fidelity transmission and also, with that, the possibility of spreading innovations. As the authors argue, such a process may help to account for the extremely long period of stasis of the Oldowan techno-complex. Technological progress in human societies seems to be achieved as long as more effective forms of communication are evolved: an increased capacity for teaching, likely implying the use of a protolanguage, must have been a prerequisite for the later development of Acheulean and Musterian lithic technologies displaying a further level of sophistication.

Already this quick and coarse-grained sketch of basic trends in Homo evolution argues for a strict entwinement between cultural (e.g., technology and teaching strategies) and biological (e.g., diet and encephalization) factors. The "gene-culture coevolution" approach has been specifically applied to patterns of human evolution (Laland et al. 2010; Gintis 2011), implying the influence of cultural processes on changes in the genetic pool of human populations. Such changes are thought of as constrained by human-specific modalities of niche construction characterized by cultural dynamics.

In this sense, we think that the causal import of the truly phenotypic level should not be overlooked. For instance, in the well-known case of adult lactose tolerance, we have a cultural dynamic (farming and dairying) that affects the distribution of alleles within a population. However, according to the main tenet of this work, the point that we would like to make here is that the primary causal dynamics, eventually bringing about changes at the level of genes in populations, are in fact a matter of phenotypes. Cultural practices can affect the phenotype of individuals, as may be clear when considering neural modifications (see also Sect. 7). For 
example, there is evidence that learning to master Palaeolithic tool-making by contemporary humans causes structural remodelling of inferior frontoparietal regions both at the grey- and at the white-matter level (Hecht et al. 2014). Other results show that adult macaque monkeys exposed to intense training in tool use (administered by human experimenters) trigger the formation of new cortico-cortical axonal branches in the intraparietal sulcus (Hihara et al. 2006; Ishibashi et al. 2002). Therefore, ascertaining whether phenotypic modifications induced by cultural dynamics, training and teaching are also accompanied by changes in the distribution of genes in populations would certainly represent a significant acquisition; yet, changes in the distribution of genes may be "just" an effect or an eventual result of phenotypic change per se, rather than the causal factors actually at play. In other words, a process of bio-cultural feedback can be proposed at the basis of human phenotypic evolution: the development of culture itself would have exerted an increasing bias on both the production of phenotypic variants and the conservation of those variants that turn out to be advantageous in the cultural context even without variation production and selection at the genetic level. Consequently, cultural phenomena have an important influence on the modalities of phenotypic variation production, spreading and stabilization along the evolution of the genus Homo and the microevolution of $H$. sapiens. The key point here is that phenotypic novelties induced by cultural practices become effective in the lifestyle and cross-generational dynamics of (human) populations before the genetic level is affected.

Moreover, the construction of human cultural niches (a key aspect in the gene-culture coevolution model) proceeds through notably different modalities in comparison to "ordinary" niche construction. Indeed, the human cultural horizon is essentially composed of practices aimed at objectives set according to human-specific, conscious representation of the external world and "abstract" planning (Amati and Shallice 2007). In a nutshell, human cultural dynamics seem to be distinctively characterized by intentional processes. Intentional processes involve the capability of consciously addressing both external reality and internal states, choosing specific goals, and accordingly behaving in accordance with deliberate courses of action. Such an intentional feature is emblematically implemented in teaching, whose progressive development throughout human prehistory and history, is both an outcome and a means of cultural evolution. Without the ability to teach, several complex cultural practices (from tool-making to industrial production, from basic rituals to institutional religious systems) could never have been established and spread. Highly sophisticated forms of teaching (such as formal schooling, compulsory education, or academia) are recent outcomes of a long process of cultural development. Moreover, teaching is intrinsically intentional in two senses: 1 ) it requires that both teacher and learner consciously and purposefully focus on the teaching process, and 2) what is transmitted and taught, the modalities of transmission, as well as the recipients of the transmitted information, are often precisely selected according to deliberately chosen priorities. All this is at the basis of the rise and stabilization of cultural traditions.

\section{Brain Plasticity and Cultural Neural Reuse}

According to the extended conception of epigenesis put forward in this paper, environmentally-induced phenotypic changes play a key role in the ways organisms cope with environmental challenges. We have also seen that such phenotypic changes may arise even independently of corresponding changes in the genomic nucleotide sequence and can be the outcome of phenotypic plasticity per se. Moreover, as said, in the case of humans, the environment is crucially constituted of cultural features as well, and those features may favour the production, spreading and conservation of suitable phenotypic variants. This seems to be 
especially evident when brain anatomy, physiology and functionality are taken into account. Indeed, brain or neural plasticity may be seen as an instance of the more general phenotypic plasticity (Sect. 2) implemented in the brain and producing changes during the whole life span of an individual (Pascal-Leone et al. 2005). Functional rearrangements of brain circuits can be triggered by repeated external stimuli deriving from cultural practices requiring specific education and training. Clearly, development represents an especially critical period in which experiences impact on the formation of the brain and of cognitive functionalities (e.g., Johnson 2001, 2011). The singularly long maturation period specific to humans is particularly relevant in this respect. Neoteny (Gould 1977, 397-404; see also Petanjek et al. 2011) refers to the fact that the human brain is significantly under-developed at birth. Although this could appear as a disadvantageous characteristic (because of the extensive and prolonged care that neotenic offspring require), it turns out to be adaptive especially in relation to a highly-social, culturallyshaped environment. The prolonged developmental period of high plasticity may allow for the gradual establishment of brain circuitries supporting human-specific cognitive functions by means of dramatic rearrangements of patterns of gene expression (Somel et al. 2009; HrvojMihic et al. 2013). Such rearrangements can in turn be triggered by environmental inputs, including, of course, cultural ones.

Importantly, the brain organization that results is still flexible enough after maturation to be partially yet remarkably re-modelled again, as a consequence of cultural practices (Changeux 2012). Neuroscientific studies have gathered evidence for use-dependent remodelling of brain anatomy occurring also beyond the developmental period (see, e.g. Draganski et al. 2006; 2014), with particular attention to training-induced modifications in the adult brain as novel skills are acquired (Draganski and May 2008).

The theoretical approach of neural reuse (Anderson 2010, 2014; Anderson and PennerWilger 2013) posits that for new cognitive functions to emerge there is no need to evolve new brain areas; on the contrary, already existing brain structures, previously evolved to subserve other functions, may be reused for the incipient new function. Neural reuse requires that the reused region is embedded in a network of other regions scattered across the cortex, so as to generate novel (functional and/or anatomical) patterns of connectivity. On the evolutionary timescale, neural reuse may be considered as a form of exaptation (Gould and Vrba 1982; Gould and Lewontin 1979; Colagè and D'Ambrosio 2014), in which rearrangements and recruitment of pre-existing brain structures may give raise to new neural networks involved in emerging functional domains. Such new networks likely derive from genetic changes enabling modified genetic-developmental programs. This might have been the case for the arcuate fasciculus - i.e. the white-matter fibre tract connecting temporal with frontal cortex in primates - that seems to have undergone significant evolution from macaques to chimpanzees to humans (Rilling et al. 2008). There is also consistent evidence that Broca's region in the inferior frontal cortex (reached by the just mentioned arcuate fasciculus, among other fibre tracts) has been reused several times in the course of primate evolution and put at the service, from time to time, of new cognitive functions: from motor tasks and tool use (Lewis 2006) to language processing (lacoboni and Wilson 2006; Vigneau et al. 2006), gestural communication (Lui et al. 2008), intention understanding (lacoboni et al. 2005), and imitation (Heiser et al. 2003). These functions did not emerge at the same time in the course of primate evolution (motor skills being, for example, much older than imitation or language), so that it is reasonable to hypothesize that Broca's area (and its evolutionary precursors) has been reused as long as new cognitive functions emerged in evolution. 
Interestingly, there seem to be cases of neural reuse that do not, and cannot, involve concomitant (or triggering) genetic evolution. These cases are often related to human cultural abilities - such as language processing, metaphorical thinking, reading or numeracy (Dehaene and Cohen 2007; Anderson 2010). Thus, the label "cultural neural reuse" could be employed (Colagè 2013; 2015; Colagè and D'Ambrosio 2014; Colagè and Oviedo 2015) to refer to a subclass of neural-reuse instances that (1) are related to cultural dynamics and (2) do not involve concomitant or triggering heritable genetic changes, in line with the proposed extended notion of epigenesis.

One of the most intriguing cases is the "visual word form area" (VWFA), a brain region specified in the left fusiform gyrus during the process of reading acquisition (Dehaene et al. 2015). Both imaging (Jobard et al. 2003; Vinckier et al. 2007) and lesion studies (Cohen et al. 2000; Gailard et al. 2006) have provided empirical evidence that VWFA's proper functioning is essential for fluent reading. There are two sets of data concerning the formation of the human brain reading system that we would like to take into account.

First, the VWFA cannot be evolved for the reading task. Indeed, writing systems were invented less than 6000 years ago (with the cuneiform system in Mesopotamia), and literacy has significantly spread across humanity only a few centuries ago (previously being a prerogative of strict minorities in human societies). On the contrary, the specification of the VWFA when an individual learns to read happens with virtually the same characteristics all around the world and irrespective of the specific writing system in use (Bolger et al. 2005; Szwed et al. 2014), even when reading is acquired in late adulthood (Dehaene et al. 2010) or when congenitally blind individuals learn to read Braille (Reich et al. 2011). There is evidence, indeed, that the portion of the left fusiform gyrus hosting the VWFA in literate individuals likely evolved for other purposes, and specifically for recognition of faces and face emotional expression (Dehaene et al. 2010; Caspers et al. 2013; 2014). This shows that exposure to the cultural dynamics of literacy induces the reuse of this left fusiform area for a new task: reading. The second point is that, consistently with what we have pointed out above (Sect. 3), acquisition of reading implies the formation of a new network subserving, as a whole, the new function (Fig. 3; see also Fig. 2). Indeed, there is evidence that the left fusiform region hosting the VWFA in literates co-activates with all the other areas of the core (spoken, articulate) language system, i.e. superior temporal (Wernicke's), inferior parietal (Geschwind's), and inferior frontal (Broca's) regions (Capers et al. 2014). Moreover, acquisition of reading induces measurable changes at the level of intrahemispheric white-matter connectivity (Yeatman et al. 2012, Thiebaut-de-Scotten et al. 2014), also in individuals who learned to read in adulthood. These changes improve the anatomical connections of the VWFA with other language areas (Fig. 3), and contribute to the formation of a new brain network specifically dedicated to reading (i.e. to link visual or tactile orthographic stimuli with phonemes and spoken words).

Summing up, the formation of a brain network for reading can be regarded as a case of cultural neural reuse in which a brain region is embedded in a new brain network and, consequently, co-opted to subserve a new function. This is supported by the functional and structural brain-imaging data reported just above. As it is highly unlikely that the human brain is "evolved for reading", we consider the formation of the brain reading network as an instance of phenotypic plasticity giving raise to a novel neural phenotype induced by exposure to a cultural environment that includes literacy. In turn, this is consistent with the extended conception of epigenesis proposed in this paper. It is also worth noting, indeed, that the case of the specification of the VWFA as an area for reading and the consequent formation of the brain 
reading system represents a good example of the organism's potentiality to produce phenotypic variations in response to environmental (in this case cultural) stimuli.

Let us add three brief considerations that will further clarify our standpoint. First, though the specification of the VWFA can be regarded as an instance of neural plasticity, it should not be equated with processes of neural (Hebbian) learning happening via local synaptic selection and strengthening. Indeed, local neural learning mainly acts by refining existing neural circuits as, likely, in the emblematic case of expansion of posterior hippocampal grey-matter in taxi drivers learning London's streets and places of interest (Woollett and Maguire 2011) - whereas we have seen that learning to read implies the formation of a new brain network connecting high-level visual regions and spoken-language areas. Second, the fact that a definite portion of the left fusiform gyrus is constantly reused to become the VWFA in literates of course depends on previous evolutionary processes that have conferred to this region working modalities and information-processing capabilities eventually suitable for reading. In the same vein, previous evolution has also supplied this region with a pattern of connectivity that predisposes it to be reused for reading (Saygin et al. 2016). However, the evolutionary processes that led to the emergence of the features that predispose the VWFA to become an area for reading had nothing to do with reading as a cognitive ability. Third, there is no way to know, at present, whether the diffusion of literacy across almost all human societies on the globe will in the future determine the spread and stabilization of genetic variants conferring specific advantages for acquiring literacy (as might happen through gene-culture coevolution processes). The key point, for us, is that - in line with the extended notion of epigenesis proposed here - ascertainable phenotypic variants induced by cultural features of human environment may precede genetic evolution, and may be effective in shaping the overall (bio-cultural) evolution of human lifeforms even in absence of corresponding genetic changes.

\section{Conclusion}

The extended notion of epigenesis put forward here is not meant to suggest a sort of allinclusive concept. We recall the proposed demarcation criteria, namely the dependence on environmental parameters plus the independence from alterations in the genetic nucleotide sequence. However, within these boundaries, it is possible to investigate and identify a substantial number of mechanisms responsible for phenotypic diversification. Not only development, but the whole life cycle of organisms is in a sense environmentally regulated, while environments in their turn are continuously modified by organisms throughout their life cycles. Intrinsic plasticity insures both a variability of responses to changing external cues, and the potential for phenotypic variants to emerge. Then, when human culture is considered, various cultural factors contributing to the environment may act on the organism, and different biological characters are exposed to the influence of those factors. Here, we turned the attention to neural modifications emerged as a consequence of certain key cultural dynamics. The notion of cultural neural reuse is introduced to deal with such cultural influences on the (human) neural phenotype, whose general characters may be expressed within the proposed extended notion of epigenesis. All this might suggest a direction of research centred on the cultural production of human phenotypes, also encompassing the influence that cultural environment can have on other biological complex traits, like metabolism or the immune system. Indeed, a more unifying picture is almost certainly yet to come and, with this aim, we think that it is highly desirable to direct attention to the causal import proper to the phenotypic dimension, where the co-construction and co-evolution of organisms and environments actually take place. 


\section{References}

Adell T, Salò E, van Loon JJWA, Auletta G (2014) Planarians Sense Simulated Microgravity and Hypergravity. Biomed Research International. doi:10.1155/2014/679672.

Allis CD, Januwein T (2016) The Molecular Hallmarks of Epigenetic Control. Nat. Rev. Genet. doi:10.1038/nrg.2016.59.

Amati D, Schallice T (2007) On the Emergence of Modern Humans. Cognition 103:358-385.

Amundson R (2005) The Changing Role of the Embryo in Evolutionary Thought, Cambridge University Press, Cambridge.

Anderson ML (2010) Neural Reuse: A Fundamental Organization Principle of the Brain. Behav. Brain Sci. 33:245-313.

Anderson ML (2014) After Phrenology: Neural Reuse and the Interactive Brain. The MIT Press, Cambridge, MA.

Anderson ML, Penner-Wilger M (2013) Neural Reuse in the Evolution and Development of the Brain: Evidence for Developmental Homology? Dev. Psychobiol. 55:42-51.

Anderson ML, Finlay BL (2014) Allocating Structure to Function: The Strong Link between Neuroplasticty and Natural Selection. Front. Human Neurosci. doi: 10.3389/fnhum.2103.00918.

Arthur W (2000) The Concept of Developmental Reprogramming and the Quest for an Inclusive Theory of Evolutionary Mechanisms. Evol. Dev. 2:49-57.

Auletta G, Colagè I, D'Ambrosio P (2015) The Game of Life Implies Both Teleonomy and Teleology. In: Sloan PR, McKenny G, Eggleson K. (eds) Darwin in the Twenty-First Century: Nature, Humanity and God. University of Notre Dame Press, Notre Dame (IN), pp. 144-163.

Auletta G, Adell T, Colagè I, D'Ambrosio P, Salò E (2012) Space Research Program on Planarian Schmidtea Mediterannea's Establishment of the Anterior-Posterior Axis in Altered Gravity Conditions. Microgravity Sci. Technol. 24:419-425.

Auletta G, Ellis GFR, Jaeger L (2008) Top-Down Causation by Information Control: From a Philosophical Problem to a Scientific Research Programme. R. Soc. Interface 5:1159-1172.

Bickerton D (2014) More than Nature Needs: Language, Mind, and Evolution. Harvard University Press: Cambridge and London.

Bolger DJ, Perfetti CA, Schneider W (2005) Cross-Cultural Effect on the Brain Revisited: Universal Structures Plus Writing System Variation. Human Brain Mapping 25:92-104.

Bouaïchi A, Roessingh P, Simpson SJ (1995) An Analysis of the Behavioural Effects of Crowding and Re-Isolation on Solitary-Reared Adult Desert Locusts (Schistocerca gregaria) and Their Offspring. Physiol. Entomol. 20:199-208.

Burggren WW (2014) Epigenetics as a Source of Variation in Comparative Animal Physiology - or - Lamarck Is Lookin' Pretty Good These Days. J. Exp. Biol. 217:682-689.

Burian RM, Gilbert SF, Mabee PM, Swalla BJ (2000) Evolutionary Developmental Biology: Paradigms, Problems and Prospects. American Zoologist 40:711-831.

Calabrese F, Rossetti AC, Racagni G, Gass P, Riva MA, Molteni R (2014) Brain-Derived Neurotrophic Factor: A Bridge between Inflammation and Neuroplasticity. Front Cell Neurosci. doi: 10.3389/fncel.2014.00430.

Caspers J, Zilles K, Eickhoff SB, Schleicher A, Mohlberg H, Amunts K. 2013. Cytoarchitectonical Analysis and Probabilistic Mapping of Two Extrastriate Areas of the Human Posterior Fusiform Gyrus. Brain Struct Funct 218:511-26. 
Caspers L, Zilles K, Amunts K, Laird AR, Fox PT, Eickhoff SB. 2014. Functional Characterization and Differential Coactivation Patterns of Two Cytoarchitectonic Visual Areas on the Human Posterior Fusiform Gyrus. Human Brain Mapping 35:2754-67.

Changeux J-P (2012) Synaptic Epigenesis and the Evolution of Higher Brain Functions. In: Sassone-Corsi P, Christen Y. (eds.) Epigenetics, Brain and Behavior, Springer-Verlag, Berlin Heidelberg, pp. 11-22.

Cohen L, Dehaene S, Naccache L, Lehéricy S, Dehaene-Lambertz G, Hénaff M-A, Michel F (2000) The Visual Word Form Area. Spatial and Temporal Characterization of an Initial Stage of Reading in Normal Subjects and Posterior Split-Brain Patients. Brain 123:291-307.

Colagè I (2013) Human Specificity: Recent Neuro-Scientific Advances and New Perspectives. ESSSAT New \& Reviews 23:5-19.

Colagè I (2015) The Human Being Shaping and Transcending Itself: Written Language, Brain, and Culture. Zygon 50:1002-1021.

Colagè I (2016) Sociality, Brain, Evolution and Culture: The Human Specificity and the Imago Dei Doctrine. Towards a Working Conceptual Framework. Studies in Science and Theology 15:4-26.

Colagè I, D'Ambrosio P (2014) Exaptation and Neural Reuse: A Research Perspective into the Human Specificity. Antonianum 89:333-358.

Colagè I, Oviedo L (2015) Cultural Influences on the Brain: Science and Theology on Human Specificity. Open Theology 1:354-365.

Collins JP, Gilbert SF, Laubichler MD, Müller GB (2007) Modelling in EvoDevo: How to Integrate Development, Evolution and Ecology. In: Laubichler MD, Müller GB (eds.) Modelling Biology. Structures, Behaviors, Evolution, The MIT Press, Cambridge (MA), pp. 355-378.

Corruccini RS (1984) An Epidemiologic Transition in Dental Occlusion in World Populations. Amer. J. Orthod. 86:419-426.

Cove DJ (2000) The Generation and Modification of Cell Polarity. J. Exp. Bot. 51:831-838.

Crawford-Young S (2006) Effects of Microgravity on Cell Cytoskeleton and Embryogenesis. Int. J. Devol. Biol. 50:183-191.

D'Ambrosio P (2016) The Epigenetic View in Life Sciences: Towards a Riper Picture of the Nature of Living Beings and Their Evolution. Studies in Science and Theology 15:195-215.

Darwin CH (1859) The Origin of Species. John Murray, London.

Darwin CH (1868) The Variation of Animals and Plants under Domestication. John Murray, London.

Daxinger L, Whitelaw E (2012) Understanding Transgenerational Epigenetic Inheritance via the Gametes in Mammals. Nat. Rev. Genet. 13:153-162.

de Vries H (1905) Species and Varieties: Their Origin by Mutation. The Open Court Publishing Company, Chicago.

Dehaene S, Cohen L (2007) Cultural Recycling of Cortical Maps. Neuron 56:384-398.

Dehaene S, Pegado F, Braga LW, Ventura P, Nunes Filho G, Jobert A, Dehaene-Lambertz G, Kolinsky R, Morais J, Cohen L (2010) How Learning to Read Changes the Cortical Networks for Vision and Language. Science 330:1359-1364.

Dehaene S, Cohen L, Morais J, Kolinsky R (2015) Illiterate to Literate: Behavioral and Cerebral Changes Induced by Reading Acquisition. Nat. Rev. Neurosci. 16:234-244.

Draganski B, May A (2008) Training-Induced Structural Changes in the Adult Human Brain. Behav Brain Res 192:137-142. 
Draganski B, Gaser C, Kempermann G, Kuhn HG, Winkler J, Büchel C, May A (2006) Temporal and Spatial Dynamics of Brain Structure Changes during Extensive Learning. J. Neurosci 26:6314-6317.

Draganski B, Kherif F, Lutti A (2014) Computational Analysis for Studying Use-Dependent Brain Plasticity. Front. Hum. Neurosci. 8:380.

Dupont C, Armant DR, Brenner CA (2009) Epigenetics: Definition, Mechanisms and Clinical Perspective. Semin Reprod Med. 27:351-357.

Edelman GM, Gally JA (2001) Degeneracy and Complexity in Biological Systems. PNAS 98:1376313768.

Ferrari PF, Tramacere A, Simpson EA, Iriki A (2013) Mirror Neurons through the Lens of Epigenetics. Trends Cog Sci 17:450-457.

Fusco G, Minelli A (2010) Phenotypic Plasticity in Development and Evolution: Facts and Concepts. Phil. Trans. R. Soc. B 365:547-556.

Gaillard R, Naccache L, Pinel P, Clémenceau S, Volle E, Hasboun D, Dupont S, Baulac M, Dehaene S, Adam C, Cohen L. 2006. Direct Intracranial, fMRI, and Lesion Evidence for the Causal Role of Left Inferotemporal Cortex in Reading. Neuron 50:191-204.

Gilbert SF (2000) Diachronic Biology Meets Evo-Devo: C.H. Waddington's Approach to Evolutionary Biology. American Zoologist 40:729-737.

Gilbert SF (2001) Ecological Development Biology: Developmental Biology Meets the Real World. Develop Biol 233:1-12.

Gilbert SF (2006) Developmental Biology. Sinauer, Sunderland (MA), ( $8^{\text {th }}$ edition).

Gilbert SF, Epel D (2009) Ecological Developmental Biology: Integrating Epigenetics, Medicine, and Evolution. Sinauer, Sunderland (MA).

Gintis H (2011) Gene-Culture Coevolution and the Nature of Human Sociality. Phil. Trans. R. Soc. B 366:878-888.

Goldberg AD, Allis CD, Bernstein E (2007) Epigenetics: A Landscape Takes Shape. Cell 128:635638.

Gould SJ (1977) Ontogeny and Philogeny, Harvard University Press, Cambridge, MA.

Gould SJ, Lewontin RC (1979) The Spandrels of San Marco and the Panglossian Paradigm: A Critique of the Adaptationist Programme Proc. R. Soc. Lond. B 205:581-598.

Gould SJ, Vrba ES (1982) Exaptation - A Missing Term in the Science of Form. Paleobiology 8:415.

Hammerstein P, Hagen EH, Herz AVM, Herzel H (2006) Robustness: A Key to Evolutionary Design. Biological Theory 1:90-93.

Hecht EE, Gutman DA, Khreisheh N, Taylor SV, Kilner J, Faisal AA, Bradley BA, Chaminade T, Stout D (2014) Acquisition of Paleolithic Toolmaking Abilities Involves Structural Remodeling to Inferior Frontoparietal Regions. Brain Struct Funct. doi: 10.1007/s00429014-0789-6.

Heiser M, lacoboni M, Maeda F, Marcus J, Mazziotta, JC (2003) The Essential Role of Broca's Area in Imitation. Eur J Neurosci 17:1123-28.

Hihara S, Notoya T, Tanaka M, Ichinose S, Ojima H, Obayashi S, Fujii N, Iriki A (2006) Extension of Corticocortical Afferents into the Anterior Bank of the Intraparietal Sulcus by Tool-Use Training in Adult Monkeys. Neuropsychologia 44:2636-2646.

Ho DH, Burggren WW (2010) Epigenetic and Transgenerational Transfer: A Physiological Perspective. J. Exp Biol 213:3-16.

Hooper LV, Bry L, Falk PG, Gordon JI (1998) Host-Microbial Symbiosis in the Mammalian Intestine: Exploring an Internal Ecosystem. BioEssays 20:336-343. 
Hooper LV, Midtvedt T, Gordon JI (2002) How Host-Microbial Interactions Shape the Nutrient Environment of the Mammalian Intestine. Annu. Rev. Nutr. 22:283-307.

Hrvoj-Mihic B, Bienvenu T, Stefanacci L, Muotri AR, Semendeferi K (2013) Evolution, Development, and Plasticity of the Human Brain. Front. Hum. Neurosci 7:707.

lacoboni M, Wilson SM (2006) Beyond a Single Area: Motor Control and Language within a Neural Architecture Encompassing Broca's Area. Cortex 42:503-506.

lacoboni M, Molnar-Szakacs I, Gallese V, Buccino G, Mazziotta JC, Rizzolatti G (2005) Grasping the Intentions of Others with One's Own Mirror Neuron System. PLoS Biology 3:e79.

Ishibashi H, Hihara S, Takahashi M, Heike T, Yokota T, Iriki A (2002) Tool-use Learning Selectively Induces Expression of Brain-Derived Neurotrophic Factor, Its Receptor trkB, and Neurotrophin 3 in the Intraparietal Multisensorycortex of Monkeys. Cogn Brain Res 14:3-9. Jablonka E, Lamb M (2006a) Evolutionary Epigenetics. In: Fox CW, Wolf JB (eds.) Evolutionary Genetics. Concepts and Case Studies, Oxford University Press, Oxford, pp. 252-264.

Jablonka E, Lamb M (2006b) Evolution in Four Dimensions. The MIT Press, Cambridge, MA. Jobard G, Crivello F, Tzourio-Mazoyer N (2003) Evaluation of the Dual Route Theory of Reading:

A Metanalysis of 35 Neuroimaging Studies. Neurolmage 20:693-712.

Johnson MH (2001) Functional Brain Development in Humans. Nat. Rev. Neurosci 2:475-485.

Johnson MH (2011) Interactive Specialization: A Domain-General Framework for Human Functional Brain Development? Develop Cogn Neurosci 1:7-21.

Kirschner M, Gerhart J (1998) Evolvability. PNAS 95:8420-8427.

Laland KN, Odling-Smee J, Myles S (2010) How Culture Shaped the Human Genome: Bringing Genetics and the Human Sciences Together. Nat Rev Genet 11:137-148.

Liebers R, Rassoulzadegan M, Lyco F (2014) Epigenetic Regulation by Heritable RNA. PLoS Genet. doi:10.1371/journal.pgen.1004296.

Lui F, Buccino G, Duzzi D, Benuzzi F, Crisi G, Baraldi P, Nichelli P, Porro CA, Rizzolatti G (2008) Neural Substrates for Observing and Imagining Non-Object-Directed Actions. Social Neuroscience 3:261-275.

Lyco F, Foret S, Kucharski R, Wolf S, Falckenhayn C, Maleszka R (2010) The Honey Bee Epigenomes: Differential Methylation of Brain DNA in Queens and Workers. PLoS Biol. doi:10.1371/journal.pbio.1000506

Manzi G (2012). Il grande racconto dell'evoluzione umana. Il Mulino, Bologna.

Margulis L, Fester R (eds) (1991) Symbiosis as a Source of Biological Innovation. The MIT Press, Cambridge, MA.

Masel J, Siegal ML (2009) Robustness: Mechanisms and Consequences. Trends Genet. 25:395403.

Mason PH (2014) Degeneracy: Demystifying and Destigmatizing a Core Concept in Systems Biology. Complexity 20:12-21.

Mazmanian SK, Liu CH, Tzlanabos AO, Kasper DL (2005) An Immunomodulatory Molecule of Symbiotic Bacteria Directs the Maturation of the Host Immune System. Cell 122:107-118.

Moczek AP, Sultan S, Foster S, Ledón-Retting C, Dworkin I, Nijhout HF, Abouheif E, Pfenning DW (2011) The Role of Developmental Plasticity in Evolutionary Innovation. Phil. Trans. R. Soc. B 278:2705-2713.

Morgan TJH, Uomini NT, Rendell RE, Chouinard-Thuly L, Street SS, Lewis HM, Cross CP, Evans C, Kearney R, de la Torre I., Whiten A, Laland KN (2015) Experimental Evidence for the CoEvolution of Hominin Tool-Making, Teaching and Language. Nature Communications 6:6029.

Müller GB (2007) Evo-Devo: Extending the Evolutionary Synthesis. Nat Rev Genet 8:943-949. 
Murray PS, Holmes PV (2011) An Overview of Brain-Derived Neurotrophic Factor and Implications for Excitotoxic Vulnerability in the Hippocampus. Intl J Peptides. doi:10.1155/2011/654085

Oyama S, Griffiths PE, Gray RD (2001) What Is Developmental Systems Theory. In: Oyama S, Griffiths PE, Gray RD (eds) Cycles of Contingencies: Developmental Systems and Evolution. The MIT Press, Cambridge MA, pp. 1-11.

Pascual-Leone A, Amedi A, Fregni F, Merabet LB (2005) The Plastic Human Brain Cortex. Annu. Rev. Neurosci. 28:377-401.

Petanjek Z, Judaš M, Šimić G, Rašin MR, Uylings HBM, Rakic P, Kostović I (2011) Extraordinary Neoteny of Synaptic Spines in the Human Prefrontal Cortex. PNAS 108:13281-13286.

Petronis A (2010) Epigenetics as a Unifying Principle in the Aetiology of Complex Traits and Diseases. Nature 465:721-727.

Pigliucci M (2009) An Extended Synthesis for Evolutionary Biology. Ann. N.Y. Acad. Sci. 1168:218-228.

Probst A, Dunleavy E, Almouzni G (2009) Epigenetic Inheritance during the Cell Cycle. Nat Rev Mol Cell Biol 10:192-206.

Ptashne M (2007) On the Use of the Word 'Epigenetics'. Curr Biol 17:R233-R236.

Reich L, Szwed M, Cohen L, Amedi A (2011) A Ventral Visual Stream Reading Center Independent of Visual Experience. Curr. Biol. 21:363-368.

Richards EJ (2006) Inherited Epigenetic Variation - Revisiting Soft Inheritance. Nat Rev Genet 7:3975-401.

Rilling JK, Glasser MF, Preuss TM, Ma X, Zhao T, Hu X, Behrens TEJ (2008) The Evolution of the Arcuate Fasciculus Revealed with Comparative DTI. Nat Neurosci 11:425-428.

Rogers SM, Matheson T, Sasaki K, Kendrick K, Simpson SJ, Burrows M (2004) Substantial Changes in Central Nervous System Neurotransmitters Accompany Phase Change in Locusts. J Exp Biol 207:3603-3617.

Saló E, Abril JF, Adell T, Cebrià F, Eckelt K, Fernandez-Taboada E, Handberg-Thorsager M, Iglesias M, Molina MD, Rodríguez-Esteban G (2009) Planarians Regeneration: Achievements and Future Direction after 20 Years of Research. Int. J. Dev. Biol. 53:1317-1327.

Sapp, J (1994) Evolution by Association. A History of Symbiosis. Oxford, Oxford University Press.

Sapp J (2003) Genesis: The Evolution of Biology. Oxford University Press, Oxford.

Sato M, Ochi T, Nakase T, Hirota S, Kitamura Y, Nomura S, Yasui N (1999) Mechanical TensionStress Induces Expression of Bone Morphogenetic Protein BMP-2 and BMP-4 but Not BMP-6, BMP-7 and GDF-5 mRNA, During Distraction Osteogenesis. J. Bone Miner. Res. 14:1084-1095.

Saygin ZM, Osher DE, Norton ES, Youssoufian DA, Beach SD, Feather J, Gaab N, Gabrieli JDE, Kanwisher N (2016) Connectivity Precedes Function in the Development of the Visual Word Form Area. Nat Neurosci. doi:10.1038/nn.4354.

Schlichting CD, Pigliucci M (1998) Phenotypic Evolution. A Reaction Norm Perspective. Sinauer, Sunderland, MA.

Schlosser G, Wagner GP (2004) The Modularity Concept in Developmental and Evolutionary Biology. In: Schlosser G, Wagner GP (eds.) Modularity in Development and Evolution, The Chicago University Press, Chicago and London, pp. 1-11.

Schick K, Toth N (2006) An Overview of the Oldowan Industrial Complex: The Sites and the Nature of Their Evidence. In: Toth N, Schick K (eds.) The Oldowan: Case Studies into the Early Stone Age, Stone Age Institute Press, Gosport, IN, pp. 3-35.

Schmalhausen II (1949) Factors of Evolution, The Blakiston Company, Philadelphia. 
Somel M, Franz H, Yan Z, Lorenc A, Guo S (2009) Transcriptional Neoteny in the Human Brain. PNAS 106:5743-5748.

Stout D (2011) Stone Toolmaking and the Evolution of Human Culture and Cognition. Phil. Trans. R. Soc. B 366:1050-1059.

Stout D, Chaminade T (2012) Stone Tools, Language and the Brain in Human Evolution. Phil. Trans. R. Soc. B 367:75-87.

Sultan SE (2007) Development in Context: The Timely Emergence of Eco-Devo. Trends Ecol. Evol. 22:575-582.

Szwed M, Qiao E, Jobert A, Dehaene S, Cohen L (2014) Effects of Literacy in Early Visual and Occipitotemporal Areas of Chinese and French Readers. J. Cogn. Neurosci. 26:45-475.

Tang GH, Rabie ABM, Hägg U (2004) Indian Hedgehog: A Mechanotransduction Mediator in Condylar Cartilage. J. Dent. Res. 83:434-438.

Thiebaut de Schotten M, Cohen L, Amemiya E, Braga LW, Dehaene S (2014) Learning to Read Improves the Structure of the Arcuate Fasciculus. Cereb Cor 24:989-95.

Tononi G, Sporns O, Edelman GM (1999) Measures of Degeneracy and Redundancy in Biological Systems. PNAS 96:3257-3262.

Varrela J (1992) Dimensional Variation of Craniofacial Structures in Relation to Changing Masticatory-Functional Demands. Eur. J. Orthod. 14:31-36.

Varrela J (2006) Masticatory Function and Malocclusion: A Clinical Perspective. Seminars in Orthodontix 12:102-109.

Vazquez-Sanroman D, Sanchis-Segura C, Toledo R, Hernandez ME, Manzo M (2013) The Effects of Enriched Environment on BDNF Expression in the Mouse Cerebellum Depending on the Length of Exposure. Behav. Brain Res. 243:118-128.

Vigneau M, Beaucousin V, Herve PY, Duffau H, Crivello F, Houde O, Mazoyer B, Tzourio-Mazoyer N (2006) Meta-Analyzing Left Hemisphere Language Areas: Phonology, Semantics, and Sentence Processing. Neuroimage 30:1414-1432.

Vinckier F, Dehaene S, Jobert A, Dubus JP, Sigman M, Cohen M (2007) Hierarchical Coding of Letter Strings in the Ventral Stream: Dissecting the Inner Organization of the Visual WordForm Area. Neuron 55:143-156.

Waddington $\mathrm{CH}$ (1942a) Canalization of Development and the Inheritance of an Acquired Character. Nature 150: 563-565.

Waddington $\mathrm{CH}$ (1942b) The Epigenotype. Endeavour 1:18-20.

Waddington $\mathrm{CH}$ (1968) The Basic Ideas of Biology. In: Waddington CH (ed) Towards a Theoretical Biology, Edinburgh, pp. 1-32.

Wagner A (2005) Robustness, Evolvability, and Neutrality. FEBS Lett. 579:1772-1778.

Walton KD, Harding S, Anschel D, Harris YT, Linás R (2005) The Effects of Microgravity on the Development of Surface Righting in Rats. J. Physiol. 565:593-608.

Waterland RA, Jirtle RL (2003) Transposable Elements: Targets for Early Nutritional Effects of Epigenetic Gene Regulation. Mol. Cell. Biol. 23:5293-5300.

Weaver ICG, Cervoni N, Champagne FA, D’Alessio AC, Sharma S, Seckl JR, Dymov S, Szyf M, Meaney MJ (2004) Epigenetic Programming by Maternal Behavior. Nat Neurosci 7:847854.

Weaver ICG (2007) Epigenetic Programming by Maternal Behavior and Pharmacological Intervention. Nature versus Nurture: Let's Call the Whole Thing Off. Epigenetics 2:22-28.

West-Eberhard MJ (2003) Developmental Plasticity and Evolution. Oxford University Press, Oxford. 
Whitacre JM (2010) Degeneracy: A Link between Evolvability, Robustness and Complexity in Biological Systems. Theoretical Biology and Medical Modelling 7:6.

Wimsatt WC, Shank JC (2004) Generative Entrenchment, Modularity, and Evolvability: When Genetic Selection Meets the Whole Organism. In: Schlosser G, Wagner GP (eds) Modularity in Development and Evolution. The Chicago University Press, Chicago and London, pp. 359-394.

Woollett K, Maguire EA (2011) Acquiring 'the Knowledge' of London's Layout Drives Structural Brain Changes. Current Biology 21:2109-2114.

Yeatman JD, Dougherty RF, Ben-Shachar M, Wandell BA (2012) Development of White Matter and Reading Skills. PNAS: E3045-E3053. 


\section{Caption of Figure 1}

Fig. 1

The extended notion of epigenesis. The notion of epigenesis (or of "epigenic" processes) is meant to encompass any process of generation of phenotypic diversification that is dependent on environmental influences and independent of changes in the genomic nucleotide sequence. Within epigenesis, epigenetics (or of epigenetic processes) denotes bio-molecular mechanisms that underlie the generation of phenotypic diversification, including those at the basis of cellular epigenetic inheritance within single organisms. Transgenerational epigenetic inheritance is in turn understood as a subset of epigenetic mechanisms ensuring the transmission of phenotypic diversification across generations of organisms via non-genetic channels. Note that not only "epigenic" processes, but also epigenetic mechanisms and mechanisms of transgenerational epigenetic inheritance should respect the two general conditions of being dependent on environmental influences and independent of changes in the genomic nucleotide sequence.

\section{Caption of Figure 2}

\section{Fig. 2}

Schematic representation of functional networks. The nodes (circles) are connected in networks that may fulfil a function. There can be different networks fulfilling the same function (e.g. Networks 1 and 2 fulfilling Function 1). Two distinct functional networks may join to constitute a new network and fulfil a third function (e.g., Networks 1 and 3 - that fulfil functions 1 and 2, respectively - joined to constitute Network 5 fulfilling Function 4; see also Fig. 3). Part of a functional network may be recruited, together with additional nodes, to fulfil an additional function (e.g., Network 4, composed of part of Network 3 and additional nodes, fulfilling Function 3).

\section{Caption of Figure 3}

\section{Fig.3}

Cultural neural reuse. Schematic representation of the formation of the brain reading system via a cultural neural reuse of the VWFA, and consequent formation of a new network out of the networks for spoken language and object recognition (ventral visual pathway). See text for more details. 
Figure 1

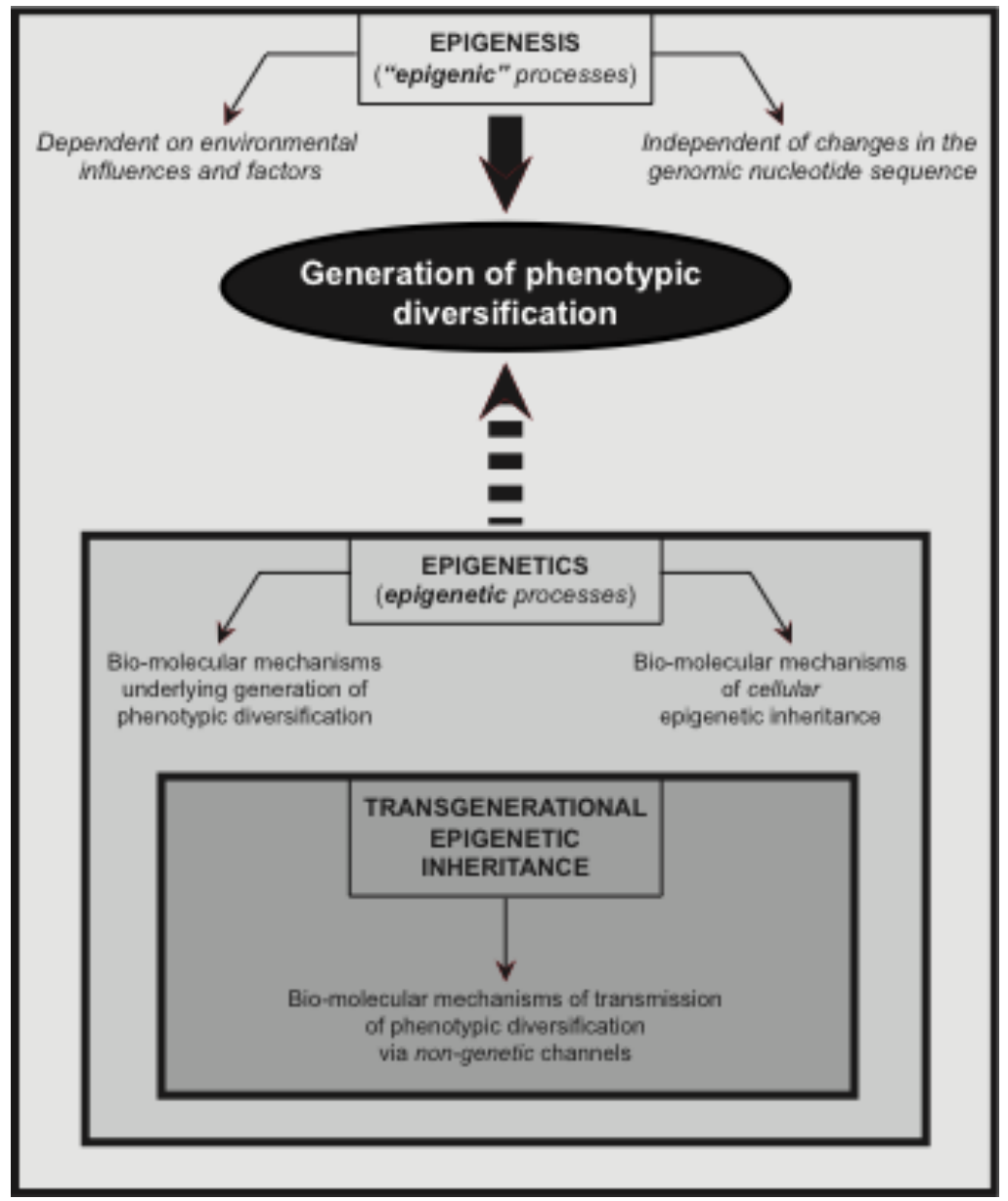


Figure 2

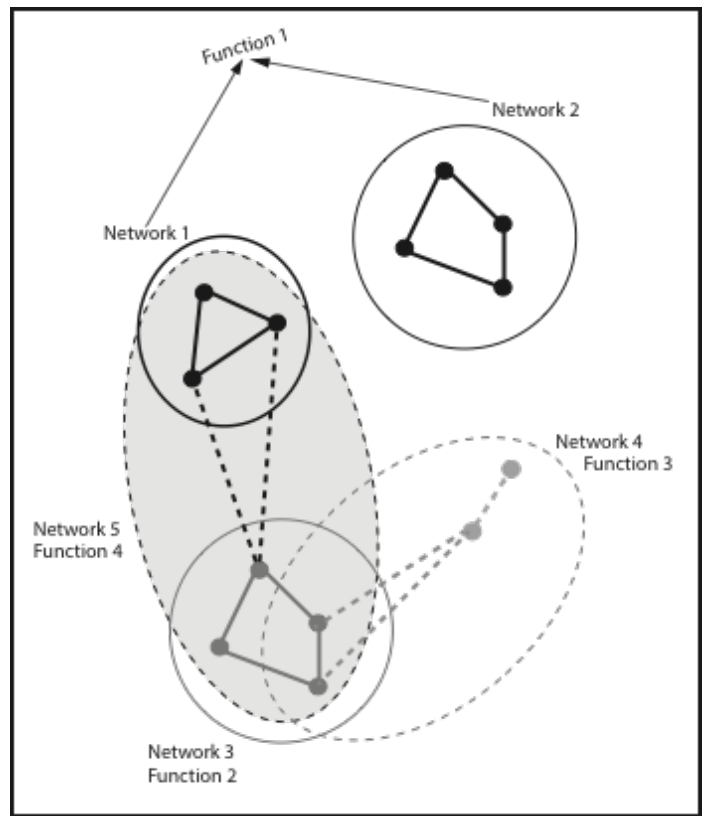

Figure 3

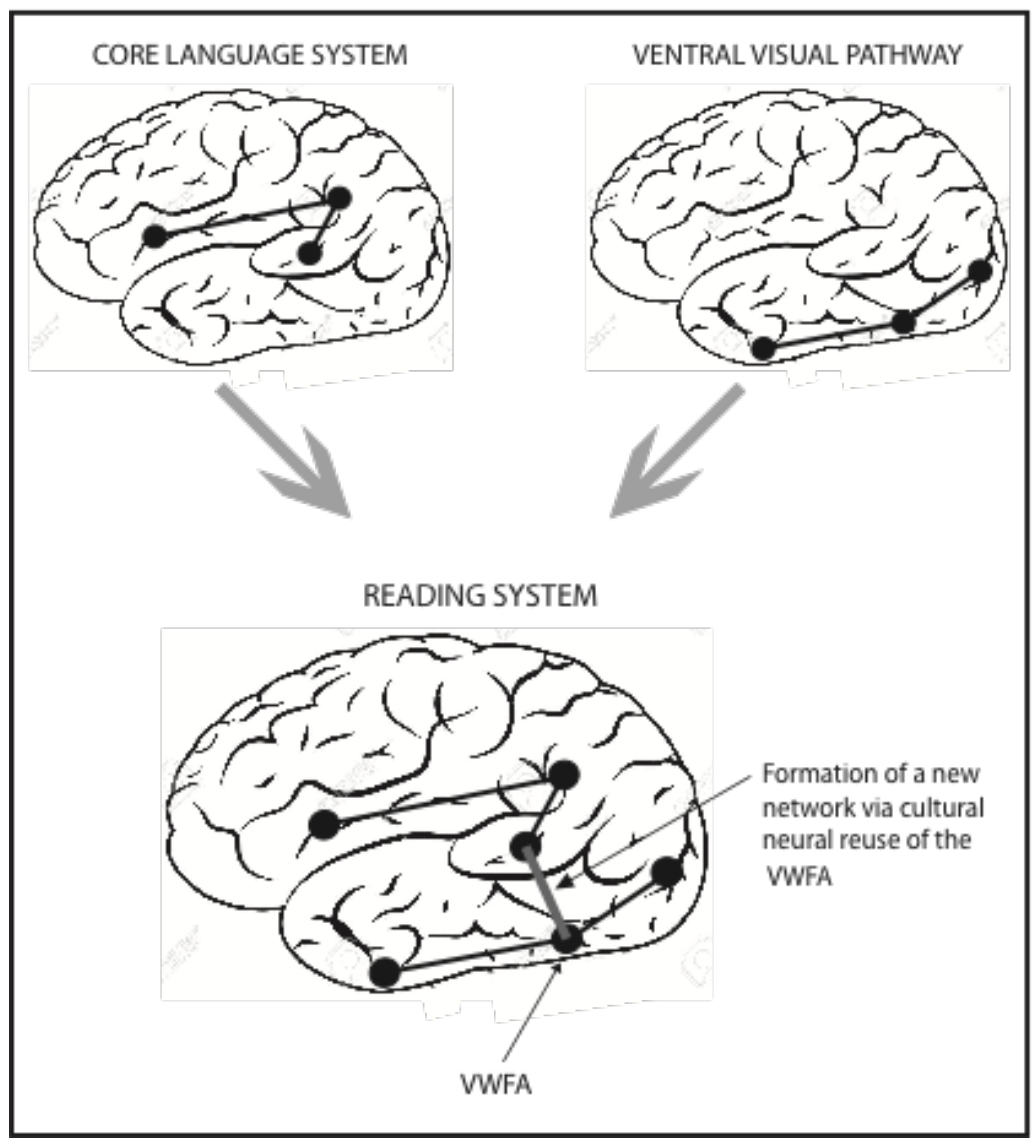

